\title{
Fall Detection Based on Sequential Modeling of Radar Signal Time-Frequency Features
}

\author{
Meng $\mathrm{Wu}^{1}$, Xiaoxiao Dai ${ }^{1}$, Yimin D. Zhang ${ }^{2}$, Bradley Davidson ${ }^{3}$, Moeness G. Amin ${ }^{2}$ and Jun Zhang ${ }^{1}$ \\ ${ }^{1}$ Department of Electrical and Computer Engineering, University of Denver, CO 80210 \\ ${ }^{2}$ Center for Advanced Communications, Villanova University, Villanova, PA 19085 \\ ${ }^{3}$ Department of Mechanical and Materials Engineering, University of Denver, CO 80210
}

\begin{abstract}
Falls are one of the greatest threats to elderly health as they carry out their daily living routines and activities. Therefore, it is very important to detect falls of an elderly in a timely and accurate manner, so that immediate response and proper care can be rendered. Radar is an effective non-intrusive sensing modality which is well suited for this purpose. It can detect human motions in all types of environments, penetrate walls and fabrics, preserve privacy, and is insensitive to lighting conditions. In this paper, we use micro-Doppler features in radar signal corresponding to human body motions and gait to detect falls using a narrowband pulse-Doppler radar. Human motions cause time-varying Doppler signatures, which are analyzed using time-frequency representations and matching pursuit decomposition for feature extraction and fall detection. The extracted features include the principal components of the time-frequency signal representations. To analyze the sequential characteristics of typical falls, we use the extracted signal features for training and testing hidden Markov models and support vector machines in different falling scenarios. Experimental results demonstrate that the proposed algorithm and method achieve fast and accurate fall detections.
\end{abstract}

Index Terms-Fall detection, Doppler radar, matching pursuit decomposition, time-frequency analysis, principal component analysis, hidden Markov model, support vector machine

\section{INTRODUCTION}

Falls of the elderly are a major public health problem as they often result in disability [1]. Falls are the main cause of accidental death in the U.S. population over age 65 [2]. Immediate assistance after a fall can reduce complications of fall risk [3]. Therefore, it is very important to detect elderly falls in a timely and accurate manner, so that immediate response and proper care can be rendered.

Many methods have bean developed for fall detection. The most common physical parameter used in fall detection is the acceleration because falling results in a variety of accelerations in different body parts. For example, by measuring the vertical acceleration of body, accelerometer devices are able to detect falls [4]. However, the drawback of such technique is that one has to wear the accelerometer devices at all time, which may prohibit daily living routines and activities.

A fall can also be monitored using non-intrusive sensory systems, for example, a radar system. Radar sensors can provide valuable human body motion. Gait characterization using various machine learning algorithms shows effective and satisfied performance [5], [6]. In [5], six features were used to represent the micro-Doppler signatures of the radar signal short-time Fourier transform (STFT) results and to train a support vector machine (SVM) for the classification of seven activities. In [6], the Doppler signatures of human activities are extracted using the mel-frequency cepstral coefficients (MFCC). Based on these features, two different machine learning algorithms, SVM and k-nearest neighbor (KNN), are then employed to detect falls. In addition, a Hidden Markov model (HMM) based machine learning approach was applied for human actions recognition in [7] based on a set of sequential images.

In this paper, we utilize the HMM to characterize the timefrequency features of radar signals for the purpose of fall detection, and the feasibility of early warning is investigated. We separately use two time-frequency analysis methods, i.e., matching pursuit decomposition (MPD) and STFT, to extract sequential feature vectors from radar signals, and the vector sequences are then transformed to symbol sequences by kmeans clustering for HMM training and testing.

The contribution of this work is threefold: 1) We propose and implement MPD that extracts sequential features from radar signals; 2) The principle component analysis (PCA) and time sequence decimation are used to extract STFT features; and 3) HMM based classification algorithms are developed and their effectiveness are verified using measurement data.

The rest of this paper consists of four sections. In Section II, we provide radar signal processing and sequential feature extraction from radar signals using MPD and STFT. The training and testing processes of HMMs are described in Section III. In Section IV, we present our experimental results and discussions. Section V concludes our research work.

\section{RADAR SIGNAL PROCESSING AND FEATURE EXTRACTION}

We denote the received narrowband radar signal in the continuous-time domain as

$$
r(t)=a(t) e^{i \theta(t)},
$$

where $a(t)$ is the amplitude, and $\theta(t)$ is the phase of the received signal.

In our experimental studies, the radar data sets were collected in the Radar Imaging Lab at the Center for Advanced Communications, Villanova University, using an Agilent network analyzer. The network analyzer was operated at carrier frequency $f_{c}=8 \mathrm{GHz}$ and was externally triggered with a 
time sampling rate of $1 \mathrm{kHz}$. The record time is 10 second for each experiment. Background subtraction is performed before the data are processed. An example of the collected radar signals is shown in Fig. 1. The periodic sampling results in discrete-time observations $r[k]=r(k T)$, where $T=10^{-3} \mathrm{~s}$ is the sampling interval.
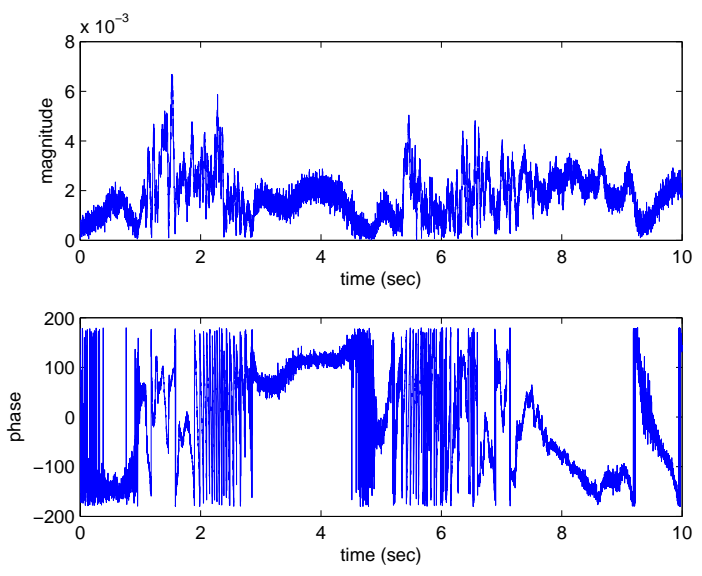

Fig. 1. Example of collected radar signal. The upper plot is the amplitude $a[k]$ and the under plot is the phase $\theta[k]$ of a received signal.

\section{A. Radar Signal Feature Extraction using Matching Pursuit Decomposition}

In this section we use the MPD algorithm to obtain the feature of the radar signal $u[k]$, which is the real part of $r[k]$, i.e., $u[k]=\Re\{r[k]\}=a[k] \cos (\theta[k])$, where $\Re\{\cdot\}$ denotes the real part operation.

The MPD algorithm decomposes the signal into a series of atoms which belong to an atom dictionary denoted by $\mathcal{D}$ [8]. Specifically, a Gaussian atom is described as

$$
g_{\varphi}[k]=\xi_{\varphi} e^{-\sigma^{2}(k T-\tau)^{2}} \cos (2 \pi v k T),
$$

where $\xi_{\varphi}$ is a normalizing constant for unit energy, $\varphi=$ $\{\tau, v, \sigma\}$ represents the time-shift, frequency-shift and scaling parameter. The signal $u[k]$ can be decomposed iteratively as

$$
u[k]=\sum_{j=0}^{J-1} \omega_{j} g_{\varphi_{j}}[k]+r_{J}[k],
$$

where $r_{J}[k]$ is the residue after $J$ MPD iterations, $r_{0}[k] \equiv$ $u[k]$. The atom used in $j$ th iteration, $g_{\varphi_{j}}[k]$, is described by parameter set $\varphi_{j}=\left\{\tau_{j}, v_{j}, \sigma_{j}\right\}$. The expansion coefficient $\omega_{j}$ is calculated as following:

$$
\omega_{j}=\sum_{k} r_{j}[k] g_{\varphi_{j}}[k], \quad j=0, \ldots, J-1 .
$$

Combining the atom parameter set $\varphi_{j}$ and atom amplitude parameter $\omega_{j}$ results in an expanded parameter vector $\mathbf{e}_{j}=$ $\left[\tau_{j}, v_{j}, \sigma_{j}, \omega_{j}\right]^{T}$ for $1 \leq j \leq J$, where $(\cdot)^{T}$ denotes matrix or vector transpose. Stacking $\mathbf{e}_{j}$ for all the $J$ iterations yields the feature sequence, denoted as $\mathbf{E}=\left[\begin{array}{llll}\mathbf{e}_{1} & \mathbf{e}_{2} \ldots \mathbf{e}_{J}\end{array}\right]$.

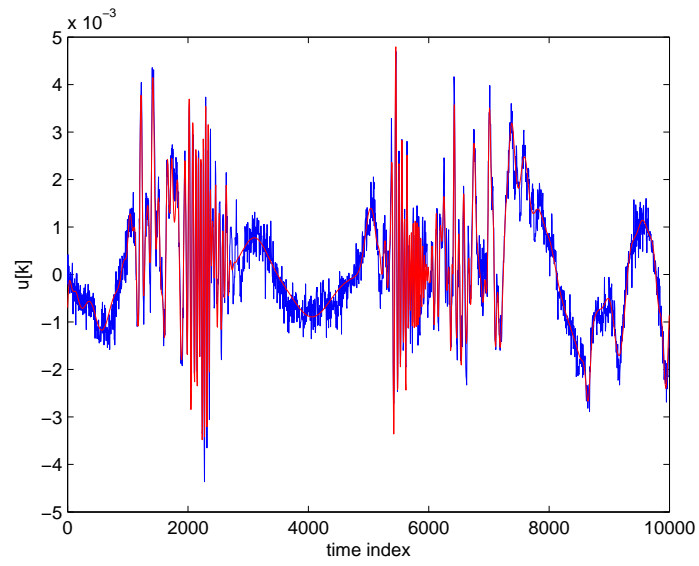

(a)

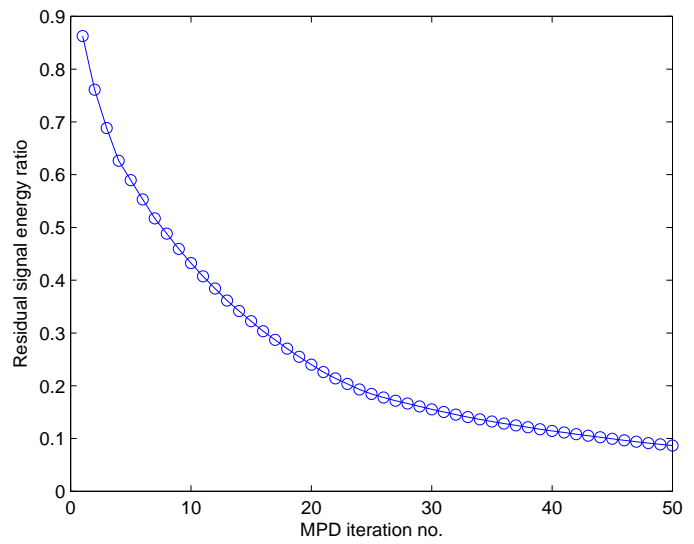

(b)

Fig. 2. Signal analysis using MPD. (a) The original signal (blue) vs. the synthesized signal (red) using 50 MPD iterations, and (b) The residual signal energy ratio after each iteration.

In Fig. 2(a), we compare an original radar signal $u[k]$ (blue curve) with its MPD representation obtained after $J=50$ iterations of decomposition (red curve). This shows that the MPD can synthesize the radar signal well. Fig. 2(b) shows that, after 50 iterations, the residual signal energy is below $10 \%$ of the original signal energy. The generated atoms are sorted in accordance with time-shift $\tau$ from small to large to form the radar signal feature vector sequence $\mathbf{E}$.

\section{B. Radar Signal Feature Extraction using STFT}

During the daily living routines and activities, velocities of different human body parts have unique patterns. For a monostatic radar, the Doppler principle states that the frequency shift of a radar signal reflected from a target is related with the target velocity as follow

$$
\Delta f=\frac{2 f_{c} \nu}{c} \cos (\alpha)
$$

where $\Delta f$ is the frequency shift, $\nu$ is the velocity of target, $c$ is the speed of light, $f_{c}$ is the carrier frequency, and $\alpha$ is 
the angle between the target moving direction and the line-ofsight direction. Because falls usually cause a high acceleration, falls and non-falls will generate different Doppler frequency patterns in received signals.

The radar signal $r[k]$ is converted into the time-frequency domain by STFT, which is given by

$$
R(m, n)=\sum_{k=1}^{K} r[k] w^{*}(k T-m T) e^{-j 2 \pi n F k},
$$

where both $m$ and $n$ are integers, $m$ is the time index, $n=$ $-200, \ldots, 0, \ldots, 200$ is the frequency index, $T=10^{-3} \mathrm{~s}$ is sampling period, $F$ is frequency step size, $w(\cdot)$ is a window function, and $(\cdot)^{*}$ denotes complex conjugate.

Because the STFT transforms one-dimensional time-domain data to two-dimensional representations in the time-frequency domain, the size of the resulting time-frequency matrix is very high. Fig. 3(a) shows the spectrogram matrix $\mathbf{S}=\left[s_{n, m}\right]$ whose $(n, m)$ th element $s_{n, m}=|R(m, n)|^{2}$ has a dimension of $400 \times 10000$, where $|\cdot|$ denotes the module operation. To facilitate HMM modeling and classification, we consider below the reduction of the matrix dimension in both time and frequency domains.

1) Dimension reduction in frequency domain: To choose a frequency range for analysis, we calculate the relative energy burst curves as [6]

$$
\begin{aligned}
& W_{1}(m)=\sum_{n=-200}^{-1} s_{n, m}^{2}+\sum_{n=1}^{200} s_{n, m}^{2}, \\
& W_{2}(m)=\sum_{n=-200}^{-25} s_{n, m}^{2}+\sum_{n=25}^{200} s_{n, m}^{2} .
\end{aligned}
$$

Comparing Figs. 3(a), 3(b) and 3(c), we find that the pattern of $W_{2}(m)$ in Fig. 3(c) is more representative for the spectrogram result depicted in Fig. 3(a). It means that the Doppler frequency ranges of -200 to $-25 \mathrm{~Hz}$ and 25 to $200 \mathrm{~Hz}$ contain more useful information. As such, spectrogram entries corresponding to Doppler frequency from -24 to $24 \mathrm{~Hz}$ are excluded from processing as they contain a high clutter energy. The spectrogram matrix $\mathbf{S}$ of dimension $400 \times 10000$ is then converted into matrix $\mathbf{P}=\left[p_{n, m}\right]$ of dimension $352 \times 10000$.

After the effective frequency range is determined, we use the PCA for feature dimension reduction. The essence of PCA is that, through an orthogonal linear transformation, data are converted to new orthogonal coordinates which ensure the variance of the projection of the data to the first coordinate to be the greatest, then the second greatest variance to the second coordinate, and so on. Those coordinates with major variances are called the principal components.

We use $\Omega$ to denote the frequency range to be used in the analysis, and $\Phi$ the new coordinate space spanned by the principal components of $\mathbf{P}$. The PCA is performed in the follows steps.

1) Calculate the mean value $z_{m}$ of the $m$ th column of $\mathbf{P}$, and subtract it from the $m$ th column. Repeating this procedure

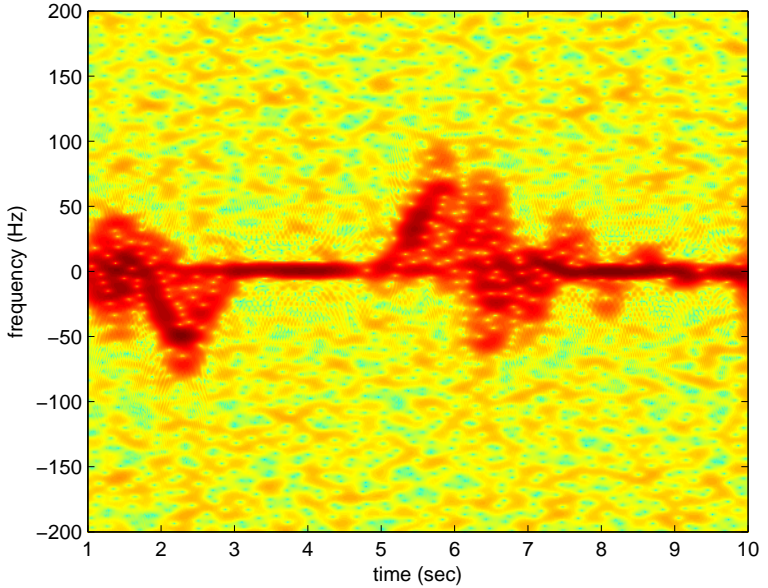

(a)

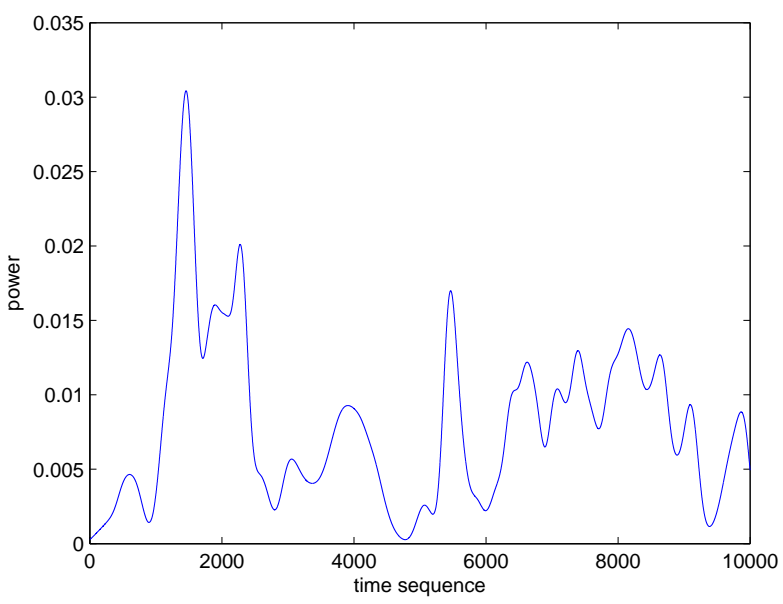

(b)

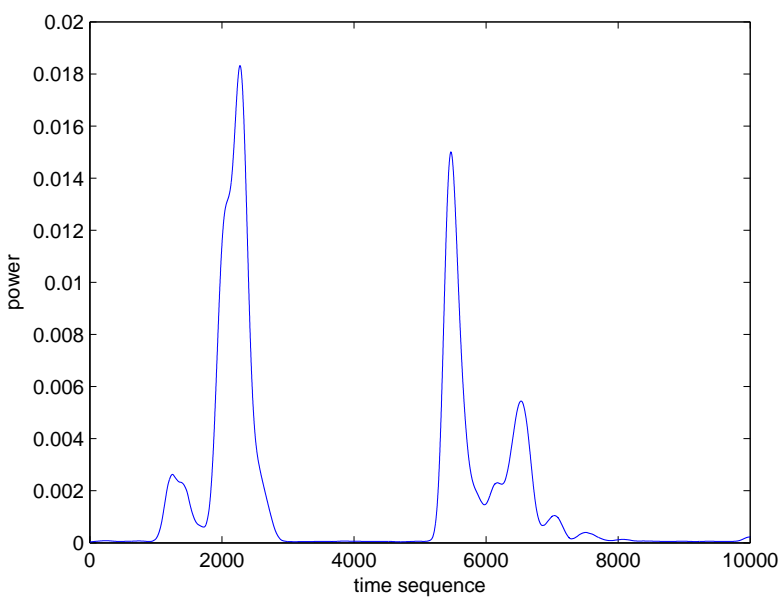

(c)

Fig. 3. Spectrogram and energy burst results of the radar data. (a) Spectrogram; (b) Energy burst curve of $W_{1}(m)$; and (c) Energy burst curve of $W_{2}(m)$. 


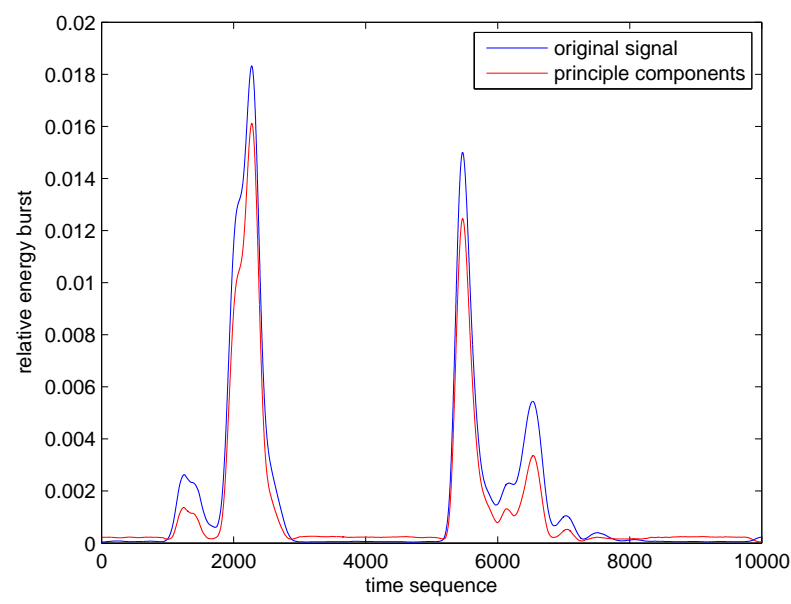

Fig. 4. Comparison of the energy bust curves.

for all the columns results in a new matrix $\mathbf{Q}=\left[q_{i, m}\right]$ with dimension $352 \times 10000$.

2) Calculate the sample covariance matrix $\mathbf{H}=\mathbf{Q} \mathbf{Q}^{T}$.

3) Calculate the eigenvalues and the corresponding eigenvectors of $\mathbf{H}$.

4) Select the first $n$ largest eigenvalues sorted in a descending order. The corresponding $n$ eigenvectors constitute a new principal component matrix $\mathbf{Y}$ with dimension $352 \times n$.

5) Calculate the projection matrix as $\mathbf{B}=\mathbf{Y}^{T} \times \mathbf{P}$, where the dimension of matrix $\mathbf{B}$ is $n \times 10^{4}$.

As a result, we are able to use principal components in space $\Phi$ to preserve the information in frequency space $\Omega$.

In Fig. 4, the energy bursts constructed from the first 10 principal components $\Phi$ (red curve) are compared with those of the original signal in $\Omega$ (blue curve). It is evident that space $\Phi$ consisting of the first 10 principal components preserves the information very well. As a result, matrix $\mathbf{P}$ of dimension $352 \times 10000$ is reduced to matrix $\mathbf{B}=\left[b_{k, m}\right]$ whose dimension is only $10 \times 10000$.

2) Dimension reduction in time-domain: Because the spectrogram transforms the one-dimensional data into a twodimensional representation, it has a higher correlation in the time-frequency domain. As such, we can decimate the spectrogram in the time domain without noticeably compromising the quality of time-frequency representations. In the following, we choose to retain 50 samples per second, which is consistent with the high speed motion camera we use. As such $\mathbf{B}$ is converted into a new matrix $\mathbf{U}=\left[\mathbf{u}_{j}\right]=\left[u_{k, j}\right]=$ $\left[b_{k, 20 j}\right]_{k=1, \ldots, 10, j=1, \ldots, 500}$ with dimensions $10 \times 500$.

After feature reduction in the frequency and time domains as described above, the spectrogram matrix $\mathbf{S}$ with dimensions $400 \times 10000$ is transformed to a feature matrix $\mathbf{U}$ with dimensions $10 \times 500$, whose columns contain the radar signal time-frequency sequences.

\section{Hidden Markov Model Based Classification}

In our study we use discrete HMMs to classify falls and non-falls. Applications of HMMs can recognize various timesequential data by automatically learning from the data sequence and optimizing model parameters [7], [9].

In our field experiment, we collected 10 data sets for each of the three motion models, i.e., sit-stand, fall backward to floor along radar radiation direction, and fall backward to floor with a $45^{\circ}$ angle to the radar radiation direction. Through the two processing methods described in Section II we obtain two types of feature sequence, $\mathbf{E}^{\alpha, \beta}$ by MPD and $\mathbf{U}^{\alpha, \beta}$ by spectrogram, where $\alpha=1,2,3$ is the model index, and $\beta=1, \ldots, 10$ is the sequence index. We use the $\mathrm{k}$-means clustering [10] to convert a feature vector sequence into a symbol sequence for HMMs.

\section{A. Model Training}

An HMM $\lambda$ is described by the following parameters as

$$
\lambda=\{\mathbf{A}, \mathbf{B}, \boldsymbol{\pi}\},
$$

where $\mathbf{A}$ is the state transition matrix, $\mathbf{B}$ is the observation probability matrix, and $\boldsymbol{\pi}$ is the initial state probability distribution vector.

Model training based on MPD features: We cluster the observation vectors generated by the MPD into 25 clusters. A feature vector, $\mathbf{e}_{j}^{\alpha, \beta}$, is transformed into a feature symbol as following:

$$
\begin{gathered}
c_{j}^{\alpha, \beta}=\arg \min _{i}\left|\mathbf{e}_{j}^{\alpha, \beta}-\mathbf{c}_{i}\right|^{2}, \\
j=1, \ldots, 50, i=1, \ldots, 25
\end{gathered}
$$

where $c_{j}^{\alpha, \beta}$ is the feature symbol corresponding to the MPD feature sequence $\mathbf{e}_{j}^{\alpha, \beta}$, and $\mathbf{c}_{i}$ is the centroid of the $i$ th cluster generated by the k-means algorithm. The feature symbol $c_{j}^{\alpha, \beta}$ is concatenated into a symbol sequence as $\mathbf{c}^{\alpha, \beta}=$ $\left[c_{1}^{\alpha, \beta}, \ldots, c_{50}^{\alpha, \beta}\right]^{T}$. We use the symbol sequence to train the corresponding model as

$$
\hat{\lambda}_{\alpha}^{\mathrm{MPD}}=\arg \max _{\lambda_{\alpha}^{\mathrm{MPD}}} \operatorname{Pr}\left(\mathbf{c}^{\alpha, \beta} \mid \lambda_{\alpha}^{\mathrm{MPD}}\right),
$$

where $\lambda_{\alpha}^{\mathrm{MPD}}$ is the HMM corresponding to the $\alpha$ th motion class. After training the HMM with the symbol sequences, we obtain the trained model parameter set $\lambda^{\mathrm{MPD}}=$ $\left\{\lambda_{1}^{\mathrm{MPD}}, \lambda_{2}^{\mathrm{MPD}}, \lambda_{3}^{\mathrm{MPD}}\right\}$.

Model training based on STFT: We cluster the observation vectors generated by the spectrogram into 50 clusters. A feature vector, $\mathbf{u}_{j}^{\alpha, \beta}$, is transformed into a feature symbol as following:

$$
\begin{gathered}
z_{j}^{\alpha, \beta}=\arg \min _{i}\left|\mathbf{u}_{j}^{\alpha, \beta}-\mathbf{z}_{i}\right|^{2}, \\
j=1, \ldots, 500, i=1, \ldots, 50
\end{gathered}
$$

where $z_{j}^{\alpha, \beta}$ is the feature symbol corresponding to the processed STFT feature sequence $\mathbf{u}_{j}^{\alpha, \beta}$, and $\mathbf{z}_{i}$ is the centroid of the $i$ th cluster generated by the k-means algorithm. The 
feature symbol $z_{j}^{\alpha, \beta}$ is concatenated into a symbol sequence as $\mathbf{z}^{\alpha, \beta}=\left[z_{1}^{\alpha, \beta}, \ldots, z_{500}^{\alpha, \beta}\right]^{T}$. We use the symbol sequences to train corresponding model as

$$
\hat{\lambda}_{\alpha}^{\text {STFT }}=\arg \max _{\lambda_{\alpha}^{\text {STFT }}} \operatorname{Pr}\left(\mathbf{z}^{\alpha, \beta} \mid \lambda_{\alpha}^{\mathrm{STFT}}\right),
$$

where $\lambda_{\alpha}^{\text {STFT }}$ is the HMM corresponding to the $\alpha$ th motion class. After training the HMM with the symbol sequences, we obtain the trained model parameter set $\lambda^{\mathrm{STFT}}=$ $\left\{\lambda_{1}^{\text {STFT }}, \lambda_{2}^{\text {STFT }}, \lambda_{3}^{\text {STFT }}\right\}$.

\section{B. Model Testing:}

Classification based on the features: The classification of the motion classes based on the MPD feature vectors is given as

$$
\hat{\alpha}=\arg \max _{\alpha} \operatorname{Pr}\left(\mathbf{c} \mid \lambda_{\alpha}^{\mathrm{MPD}}\right),
$$

where $\mathbf{c}$ is a feature sequence from an unknown motion class.

Classification based on STFT: the classification of the motion classes based on the STFT feature vectors is given as

$$
\hat{\alpha}=\arg \max _{\alpha} \operatorname{Pr}\left(\mathbf{z} \mid \lambda_{\alpha}^{\mathrm{STFT}}\right),
$$

where $\mathbf{z}$ is a feature sequence from an unknown motion class.

In this paper, we also present results of early fall detection, where we use the first section of the feature sequence with various lengths to predict and detect falls.

\section{EXPERIMENTAL RESULTS AND DISCUSSION}

The radar signal data are collected using a narrowband pulse-Doppler radar implemented using a network analyzer, which is operated at a carrier frequency of $8 \mathrm{GHz}$. The network analyzer is triggered at a $1 \mathrm{kHz}$ sampling rate, and the record time of each experiment is 10 seconds. We build and test the HMM algorithm based on the following human motion classes: 1) repeated sitting and standing, 2) falling backward to floor along the radar radiation direction, and 3) falling backward to floor with a $45^{\circ}$ angle to the radar radiation direction. Each category of motion class has 10 experimental data sets.

Tables I and II demonstrate the confusion matrix of the HMM based classification by respectively exploiting the MPD and STFT features. In Table I, the element of the confusion matrix corresponding to $\mathbf{F}^{i, \beta}$ and $\lambda_{j}^{\mathrm{MPD}}$ represents the ratio of the feature vectors from motion class $i$ being classified into class $j$. The entries in Table II are similarly defined. Tables I and II clearly show that both MPD and STFT extract the features sequences for the HMM-based methods to yield reliable classification results.

TABLE I

HMM RECOGNITION RATE BASED ON MPD FEATURES

\begin{tabular}{|c|c|c|c|}
\hline & $\lambda_{1}^{\mathrm{MPD}}$ & $\lambda_{2}^{\mathrm{MPD}}$ & $\lambda_{3}^{\mathrm{MPD}}$ \\
\hline $\mathbf{F}^{1, \beta}$ & $90 \%$ & 0 & $10 \%$ \\
\hline $\mathbf{F}^{2, \beta}$ & 0 & $100 \%$ & 0 \\
\hline $\mathbf{F}^{3, \beta}$ & 0 & $10 \%$ & $90 \%$ \\
\hline
\end{tabular}

TABLE II

HMM RECOGNITION RATE BASED ON STFT FEATURES

\begin{tabular}{|c|c|c|c|}
\hline & $\lambda_{1}^{\text {STFT }}$ & $\lambda_{1}^{\text {STFT }}$ & $\lambda_{1}^{\text {STFT }}$ \\
\hline $\mathbf{U}^{1, \beta}$ & $100 \%$ & 0 & 0 \\
\hline $\mathbf{U}^{2, \beta}$ & 0 & $100 \%$ & 0 \\
\hline $\mathbf{U}^{3, \beta}$ & $10 \%$ & 0 & $90 \%$ \\
\hline
\end{tabular}

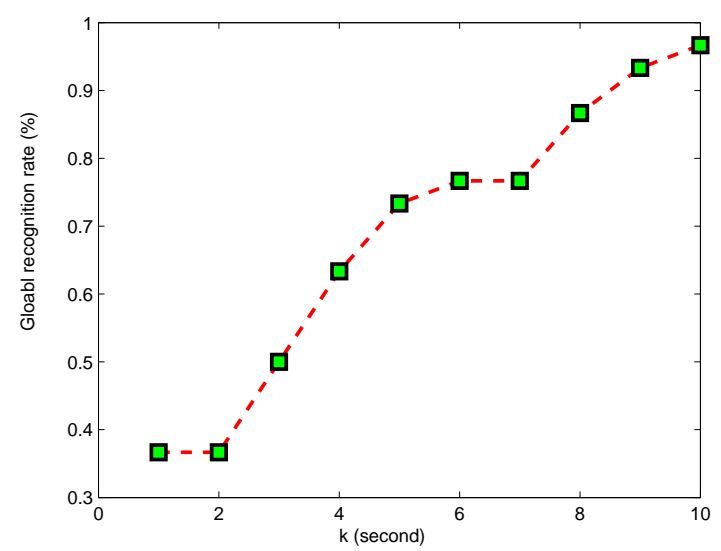

Fig. 5. The global recognition rate using initial parts of the feature symbol sequence.

Next, we explore the early fall detection with the observation sequences $\mathbf{U}^{\alpha, \beta}$ extracted from the spectrogram. From the 10 -second data in each collected data set, we separately use the first $k$ seconds to study the classification performance. The global recognition rate is defined as

$$
c(k)=\frac{N_{c}(k)}{N},
$$

where $N_{c}(k)$ is the number of correct recognitions using the first $k$ seconds of data, and $N$ is the total number of recognitions. The results are shown in Fig. 5 as a function of $k$.

We regard the entire 10 seconds of the radar recording as an event, and use the early information of an event to forecast which motion is going to take place. As we can see from Fig. 5, the results improve as more number of observations of an event becomes available. An $75 \%$ forecast accuracy is achieved using the information of the first 5 seconds, whereas the accuracy is increased to $85 \%$ when the first 8 seconds of the observed data are used.

\section{Support Vector Machine Based Classification}

In this section, we present a brief introduction of SVM, which is widely applied for classification and regression analysis. The form of SVM's training data can be denoted as $G=$ $\left\{\left(\mathbf{x}_{i}, y_{i}\right), i=1, \ldots, M\right\}$, where $\mathbf{x}_{i}=\left[x_{i, 1}, x_{i, 2}, \ldots, x_{i, m}\right]^{T} \in$ $\Re^{m}$ is a feature vector and $y_{i} \in\{+1,-1\}$ denotes a class label of the vector $\mathbf{x}_{i}$. Kernel-based SVM can separate the set of training data $G$ into two classes by a hyperplane. In our paper, we use the the C-SVM model to find the optimal hyperplane 
by solving the following minimization problem [11]:

$$
\begin{array}{ll}
\min _{\mathbf{w}, b, \xi_{i}, i=1, \ldots, M} & \frac{1}{2} \mathbf{w}^{T} \mathbf{w}+C \sum_{i=1}^{M} \xi_{i} \\
\text { subject to } & y_{i}\left(\mathbf{w} \cdot \mathbf{x}_{i}\right)+b \geq 1-\xi_{i}, \\
& \xi_{i} \geq 0,
\end{array}
$$

where $\mathbf{w}$ is the direction vector of a optimal separation hyperplane, $C>0$ denotes the penalty parameter of the error term, and $\xi_{i}, i=1, \ldots, M$, are slack variables. Minimizing the object function yields the optimal separation hyperplane. Thus, for test data vector $\mathbf{x}$, the decision function is expressed as

$$
\sum_{i \in Z} y_{i} \alpha_{i}\left\langle\mathbf{x} \cdot \mathbf{x}_{i}\right\rangle+b=0
$$

where $Z$ is a subset of the indices $\{1, \ldots, M\}$, which means that not all of training data $G$ can be the support vectors, and $\alpha_{i}$ is the Lagrangian multiplier. Substituting $\left\langle\mathbf{x} \cdot \mathbf{x}_{i}\right\rangle$ by the kernel function $K\left(\mathbf{x}, \mathbf{x}_{i}\right)$ in the above expression results in the following decision function:

$$
f(\mathbf{x})=\operatorname{sgn}\left(\sum_{i \in Z} y_{i} \alpha_{i} K\left(\mathbf{x}, \mathbf{x}_{i}\right)+b\right) .
$$

We use the MPD features of the radar signal as the training and testing data for the SVM. The MPD feature vector can be denoted as a $1 \times 200$ row vector $\mathbf{x}_{i}=$

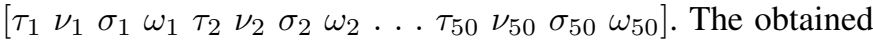
results are summarized in TABLE III with type 1 denoting the repeated sitting and standing, type 2 falling backward to floor along the radar radiation direction, and type 3 falling backward to floor with a $45^{\circ}$ angle to the radar radiation direction.

TABLE III

SVM RECOGNITION RATE BASED ON MPD FEATURES

\begin{tabular}{|c|c|c|c|}
\hline & type 1 & type 2 & type 3 \\
\hline type 1 & $100 \%$ & 0 & 0 \\
\hline type 2 & 0 & $100 \%$ & 0 \\
\hline type 3 & 0 & 0 & $100 \%$ \\
\hline
\end{tabular}

\section{CONCLUSIONS}

In this paper, novel fall detection techniques were developed based on time-frequency analysis and hidden Markov models (HMMs). We first presented two methods, the matching pursuit decomposition and the spectrogram, for extracting features from radar signals reflected by human bodies to detect falls. Based on these features, HMM based classification approaches were applied. The processed results show that the proposed approach is very effective in detecting and classifying human falls. We also proposed early forecast for a fall event and demonstrated promising results. The support vector machine (SVM) recognition rate based on matching pursuit decomposition (MPD) features shows that the SVM is also a very promising method.

\section{ACKNOWLEDGMENT}

The authors would like to thank Dr. Fauzia Ahmad, Director of the Radar Imaging Lab at the Center for Advanced Communications, Villanova University, for kindly providing the lab facility and helping the data collection.

\section{REFERENCES}

[1] S. Sadigh, A. Reimers, R. Andersson, and L. Laflamme, "Falls and fall related injuries among the elderly: a survey of residential-care facilities in a swedish municipality," Journal of Community Health, vol. 29, pp. 129-140, 2004.

[2] S. L. Murhy, "National vital statistics reports," Tech. Rep., National Center for Health Statistics, 2000.

[3] C. G. Moran, R. T. Wenn, M. Sikand, and A. M. Taylor, "Early mortality after hip fracture: is delay before surgery important," Journal of Bone and Joint Surgery, vol. 87, pp. 483-489, 2005.

[4] D. Giansanti, G. Maccioni, and V. Macellari, "The development and test of a device for the reconstruction of 3-D position and orientation by means of a kinematic sensor assembly with rate gyroscopes and accelerometers," IEEE Transactions on Biomedical Engineering, vol. 52, pp. 1271-1277, 2005.

[5] Y. Kim and H. Ling, "Human activity classification based on microdoppler signatures using a support vector machine," IEEE Transactions on Geoscience and Remote Sensing, vol. 47, pp. 1328-1337, 2009.

[6] L. Liu, M. Popescu, M. Skubic, M. Rantz, T. Yardibi, and P. Cuddihy, "Automatic fall detection based on Doppler radar motion signature," in Proceedings of International Conference on Pervasive Computing Technologies for Healthcare, 2011.

[7] J. Yamato, J. Ohya, and K. Ishii, "Recognizing human action in timesequential images using hidden Markov model," in Proceeding of IEEE Computer Society Conference on Computer Vision and Pattern Recognition, 1992.

[8] S. G. Mallat and Z. Zhang, "Matching pursuits with time-frequency dictionaries," IEEE Transactions on Signal Processing, vol. 41, pp. 3397-3415, 1993.

[9] L. R. Rabiner, "A tutorial on hidden Markov models and selected applications in speech recognition," Proceedings of the IEEE, vol. 77, pp. 257-286, 1989.

[10] T. Kanungo, D. M. Mount, N. S. Netanyahu, C. D. Piatko, R. Silverman, and A. Y. Wu, "An efficient k-means clustering algorithm: analysis and implementation," IEEE Transactions on Pattern Analysis and Machine Intelligence, vol. 24, pp. 881-892, 2002.

[11] C. J. Lin, "A formal analysis of stopping criteria of decomposition methods for support vector machines," IEEE Transactions on Neural Networks, vol. 13, pp. 1045-1052, 2002. 